\title{
Estilos de negociación de conflicto y satisfacción sexual en parejas adventistas de Lima Metropolitana
}

\author{
Roxana Quilla-Varga* \\ Departamento de Psicología, I.E Particular San Antonino de Carapongo Calle 16 Mz V Lote 39, Chosica, Lima
}

\section{RESUMEN}

Objetivo: Determinar la relación entre los estilos de negociación del conflicto y la satisfacción sexual. Material y Métodos: El estudio corresponde al diseño no experimental, transversal de tipo correlacional. La población está constituida por personas casadas de la religión adventista cuyas edades fluctúan entre 24 y 75 años. El tamaño de la muestra se estimó con procedimientos no probabilísticos ascendiendo a 67 sujetos. Se aplicó el Inventario de Estilos de negociación del conflicto de Levinger y Pietromonaco que cuenta con 30 ítems y mide cuatro estilos; asimismo, se aplicó el Inventario de satisfacción sexual de Álvarez-Gayou, Honold y Millán, con 29 ítems y clasificada en tres niveles. Resultados: Se encontró que existe algún tipo de asociación $(\mathrm{F}=4.793, \mathrm{p}<0.05)$ entre la variable dependiente (satisfacción sexual) y la variable independiente (estilos de negociación del conflicto). El estilo de resolución de conflicto de colaboración y evitación tiene relación significativa con la satisfacción sexual $(\mathrm{r}=0.441, p<0.01 ; \mathrm{r}$ $=0.235, p<0.05)$; mientras que el estilo de acomodación y de competencia no están asociados con la satisfacción sexual ( $\mathrm{r}=0.055, p>0.01 ; \mathrm{r}=-0.032, p>0.01)$. Conclusiones: Ambas variables de estudio tienen algún tipo de relación. Específicamente el estilo de colaboración y de evitación se correlaciona positivamente con la satisfacción sexual; a diferencia de los estilos acomodación y competencia que no presentan relación con la satisfacción sexual.

Palabras clave: Negociación, resolución, conflicto, colaboración, acomodación, evitación, competencia, satisfacción sexual.

\begin{abstract}
Objective: Determine the relationship between conflict negotiation styles and sexual satisfaction. Materials and Methods: The design is not experimental, Cross-sectional study and correlational style. The population is constituted for person's married Adventist denomination whom ages range between 24 and 75 years. The sample of the population in studies was estimated using non-probability procedures amounting to 67 subjects. It used The Negotiation Styles Inventory of Conflict and of Levinger and Pietromonaco which has 30 items and measures four styles; and the Sexual Satisfaction Inventory from Alvarez-Gayou, Honold and Millan, with 29 items, and classified into three levels. Results: It has been found that the study variables are associated $(\mathrm{F}=4.793, \mathrm{p}<0.05)$. The style of conflict resolution collaboration and Avoidance has significant relationship with sexual satisfaction sexual $(\mathrm{r}=$ $0.441, p<0.01 ; \mathrm{r}=0.235, p<0.05)$; while the styles accommodation and competition are not associated with sexual satisfaction $(\mathrm{r}=0.055, p>0.01 ; \mathrm{r}=-0.032, p>0.01)$. Conclusions: Both study variables have some relationshi Specifically the collaborative style and avoidance is positively correlated with sexual satisfaction, as opposed to accommodation and competition styles have no relation to sexual satisfaction.
\end{abstract}

Keywords: Negotiation, resolution, conflict, collaboration, accommodation, avoidance, competition, sexual satisfaction. 


\section{INTRODUCCIÓN}

Este tema de investigación sitúa la problemática en las parejas adventistas. Consideramos que algunos cónyuges que pertenecen a la iglesia adventista tienen serias dificultades en la forma de resolver los conflictos y en la satisfacción sexual; y estos problemas se acrecientan más en la vida marital. Cuando un matrimonio se establece, los cónyuges muchas veces no consideran las pautas para resolver los conflictos, la información adecuada sobre la satisfacción sexual y emocional, el presupuesto familiar, el estilo de crianza de los hijos, las pautas de convivencia y la relación con los familiares políticos. Esto permite que los cónyuges vivan en un ambiente de inseguridad, crisis marital, precediendo una posible separación y/o divorcio. En el Perú, con la ley 29227 se registraron 12,696 divorcios con una tasa anual creciente de 3.9\%. En Lima, el Cono Centro tiene el mayor índice con $63,9 \%$, seguido del Cono Sur con $14,2 \%$ de divorcios, donde pertenecen los distritos en estudio (veinticuatro municipios de Lima, 2010; Instituto Nacional de Estadística e Informática, 2007). Las denominaciones cristianas tampoco son inmunes. Los bautistas alcanzan la tasa mayor de divorcios y en los adventistas se estima que uno de cada cuatro termina en divorcio (Wicker, 2000; Rowe, 2007).

En el matrimonio los conflictos y las discusiones son inevitables, el asunto es si están preparados para resolver el conflicto con un estilo adecuado (Chico, 2010); cuando se desconocen de cómo manejar las diferencias con la pareja, entonces se produce el divorcio (Vallejo, Sánchez y Sánchez, 2004; Rowe, 2007). La resolución de conflicto busca estrategias para descubrir la raíz del problema y eliminar el conflicto (Arana, 2002); existen cuatro estilos para resolver el conflicto: colaboración, acomodación, evitación y competencia. Por ejemplo las investigaciones mexicanas de Flores, Díaz y Rivera (2004), Flores, Díaz, Rivera y Chi (2005) encontraron que el estilo más utilizado para resolver los conflictos es el de colaboración. Asimismo, hallaron que las mujeres son flexibles; algunas veces se someten a los deseos de su pareja y, en otras circunstancias, imponen su punto de vista saliéndose con la suya.

Cuando las necesidades sexuales y emocionales del hombre y de la mujer no son satisfechas; el matrimonio tarde o temprano fracasa (Núñez, 2007; Van Pelt, 2004). La satisfacción sexual se entiende por una evaluación subjetiva de agrado o desagrado que realiza una persona respecto a su vida sexual. La tarea del terapeuta es capacitar en el manejo del conflicto e intervenir en los problemas sexuales que conllevan al divorcio (Gilbert \& Shmukler, 2000; Ortiz, 1999).
Rosberg y Rosberg (2006) refieren que cuando hay "Brechas abiertas" o conflictos no resueltos adecuadamente, se convierte en una barrera para logar la satisfacción sexual, manifestándolo de la siguiente manera: "cuando esposo y esposa discuten y tienen conflictos, les resulta difícil hacer a un lado sus diferencias y saltar a la cama" (176). Asimismo, Álvarez-Gayou (1986) menciona que las parejas que tienen conflictos también tienen problemas en la relación erótica; lo cual genera insatisfacción sexual.

En la investigación que realizó Zavala (2009) en la ciudad de Lima encontró que $51 \%$ de la población tiene insatisfacción sexual, a diferencia de las poblaciones mexicanas que gozan de una buena satisfacción sexual (Álvarez-Gayou, Honold y Millán, 2005; Honold, 2006). Por lo tanto, el objetivo de esta investigación es determinar si existe relación entre los estilos de negociación de conflicto y la satisfacción sexual en parejas adventistas de los distritos misioneros de Surquillo y Villa María "A" en Lima Metropolitana. Esta investigación contribuye al conocimiento científico y eclesiástico.

Asimismo, permite tener una prueba de validez y confiabilidad de los instrumentos de recolección de datos. Consideramos que este trabajo de investigación será de mucho beneficio para las parejas adventistas e investigadores porque no se han encontrado estudios similares en la población, ya que este conocimiento dará apertura al desarrollo y fortalecimiento conyugal, búsqueda profesional para el manejo de conflictos, pautas para lograr la satisfacción sexual, sabiendo que el matrimonio es la base de una sociedad. Asimismo, se espera contribuir en el bienestar de los matrimonios de la población adventista, de modo que se puedan realizar programas de intervención, mejorando la eficacia en la resolución de conflictos; así como en la satisfacción sexual.

\section{MATERIAL Y MÉTODOS}

Esta investigación es de diseño no experimental de tipo correlacional y transversal. La muestra estuvo conformada por 67 personas de ambos géneros, se utilizó el muestreo no probabilístico del tipo por conveniencia, donde se aplicó los instrumentos cumpliendo los criterios de inclusión y exclusión.

El primer instrumento es el inventario de Negociación de Conflicto de Levinger y Pietromonaco (1989), originalmente con 31 ítems y fue aplicado por primera vez en México por Sánchez (2000) y la validez factorial fue realizada por Rivera (2000) y 
Rivera, Díaz y Sánchez, (2002); Sin embargo, para esta investigación se ejecutó el proceso de adaptación del instrumento.

La validez de contenido se realizó por criterio de jueces expertos y la validez de constructo mediante la técnica de análisis de sub test-test e ítem-sub test. Asimismo, se encontró que este inventario tiene una alta confiabilidad de 0.823 alfa de Cronbach.

Este instrumento cuenta con 30 ítems, una escala de likert e incluye cuatro factores que miden cuatro estilos: Colaboración, Acomodación, Evitación y competencia.

El otro instrumento que se utilizó fue el Inventario de Satisfacción Sexual elaborado el doctor Juan Luis Álvarez-Gayou Jurgenson y colaboradores del Instituto Mexicano de Sexología. Para los fines de esta investigación se ejecutó el proceso de adaptación, obteniéndose una confiabilidad de alfa de Cronbach de 0.8737; asimismo, presenta validez de contenido por jueces expertos y validez de constructo, encontrándose en todos sus factores y correlaciones muy significativas oscilando entre 0.774 y 0.965 al $\mathrm{p}<0.01$. Consta de 29 ítems con una escala de Likert y tiene seis factores: Afectividad, Bienestar, Erotismo, Respeto y responsabilidad, Estímulos sensoriales y Comunicación. Asimismo, se clasifica en tres niveles de satisfacción sexual: buena, regular y mala.

Para la recolección de datos se coordinó con los líderes eclesiásticos de la Iglesia Adventista del Séptimo Día (IASD): pastores distritales, directores de las congregaciones, ancianos de las iglesias adventistas que dirigen los distritos misioneros de Surquillo y Villa María "A" de Lima Metropolitana. Los instrumentos de aplicación fueron de forma individual y colectiva. Una vez obtenidos los inventarios, el análisis se realizó utilizando el software estadístico SPSS 15.0, realizando las pruebas estadísticas como la regresión lineal múltiple y la Correlación de Pearson. Posteriormente se presentó los resultados en tablas, cumpliendo con los objetivos de la investigación.

\section{RESULTADOS}

Tabla 1

Correlación de los estilos de negociación de conflicto y satisfacción sexual, mediante el análisis de regresión lineal múltiple.

\begin{tabular}{ccccc}
\hline Modelo & $\mathrm{R}$ & R cuadrado & R cuadrado corregida & $\begin{array}{l}\text { Error tí de la } \\
\text { estimación }\end{array}$ \\
\hline 1 & $.486(\mathrm{a})$ & .236 & .187 & 22.16515 \\
\hline
\end{tabular}

En la tabla 1 se muestra el coeficiente de correlación $\mathrm{R}$ y el coeficiente de determinación $\mathrm{R}^{2}$. El valor $\mathrm{R}=$ 0.486 indica que existe una relación lineal directa $\mathrm{y}$ moderada entre las variables. El valor $\mathrm{R}^{2}=0.236$ nos indica que las variables predictores de los estilos de negociación del conflicto (colaboración, acomodación, evitación y competencia) explican el $23.6 \%$ de la variabilidad en la manifestación de la satisfacción sexual.

Tabla 2

Análisis de regresión lineal múltiple para la variable dependiente de satisfacción sexual.

\begin{tabular}{cccccc}
\hline & $\begin{array}{c}\text { Suma de } \\
\text { cuadrados }\end{array}$ & Gl & $\begin{array}{c}\text { Media } \\
\text { cuadrática }\end{array}$ & F & Sig. \\
\hline Regresión & 9418.454 & 4 & 2354.613 & 4.793 & $.002(a)$ \\
Residual & 30460.233 & 62 & 491.294 & \\
Total & 39878.687 & 66 & & \\
\hline $\begin{array}{l}\text { a Variables predictoras: (constante), estilo competencia, estilo evitación, estilo acomodación, estilo } \\
\text { colaboración } \\
\text { b Variable dependiente: satisfacción sexual }\end{array}$
\end{tabular}


En la tabla 2 se puede observar que el resultado del análisis de la varianza del modelo de regresión lineal múltiple es significativo $(\mathrm{F}=4.793, \mathrm{p}<0.05)$. Por lo tanto, rechazamos la hipótesis nula de que la variabilidad observada en la variable satisfacción

Tabla 3

Análisis de correlación entre el estilo de colaboración y la satisfacción sexual. sexual sea explicada por el azar, y admitimos que hay algún tipo de asociación entre la variable dependiente (satisfacción sexual) y las variables independientes (colaboración, acomodación, evitación y competencia).

\begin{tabular}{|c|c|c|}
\hline \multirow{3}{*}{ Satisfacción sexual } & \multicolumn{2}{|c|}{ Estilo Colaboración e igualdad } \\
\hline & $r$ & $p$ \\
\hline & $0.441 * *$ & 0.000 \\
\hline
\end{tabular}

\footnotetext{
**. La correlación es significativa al nivel 0,01
}

En la tabla 3 se evidencia que el estilo de correlación moderada de forma significativa $(r=0.441$; colaboración y la satisfacción sexual tienen una $p<0.01)$ indicando que ambas variables tienen relación.

Tabla 4

Análisis de correlación entre el estilo de acomodación y satisfacción sexual.

\begin{tabular}{lll}
\hline & \multicolumn{3}{c}{ Estilo Acomodación } \\
\cline { 2 - 3 } Satisfacción sexual & $r$ & $p$ \\
\cline { 2 - 3 } & 0.055 & 0.660 \\
\hline
\end{tabular}

En la tabla 4 se observa que la correlación entre el estilo de acomodación y la satisfacción sexual es

Tabla 5

Análisis de correlación entre el estilo de evitación y la satisfacción sexual.

positiva no significativa $(\mathrm{r}=0.055 ; p>0.01)$, reflejando que ambas variables no tienen relación.

\begin{tabular}{lll}
\hline & \multicolumn{2}{c}{ Estilo Evitación } \\
\cline { 2 - 3 } Satisfacción sexual & $r$ & $p$ \\
\cline { 2 - 3 } & $0.235^{*}$ & 0.028 \\
\hline
\end{tabular}

\footnotetext{
*. La correlación es significativa al nivel 0,05
}

En la tabla 5 podemos ver que el estilo evitación y la satisfacción sexual tiene una correlación baja

Tabla 6

Análisis de correlación entre el estilo de competencia y la satisfacción sexual.

\begin{tabular}{lcc} 
& \multicolumn{3}{c}{ Estilo Competencia } \\
\cline { 2 - 3 } Satisfacción sexual & $r$ & $p$ \\
\cline { 2 - 3 } & -0.032 & 0.797
\end{tabular}

significativa $(r=0.235 ; p<0.05)$, indicando que ambas variables tienen relación. 
En la tabla 6 percibimos que la correlación entre el estilo de competencia y satisfacción es negativa no significativa $(r=-0.032 ; p>0.01)$, mostrando que ambas variables no tienen relación.

\section{DISCUSIÓN}

Muchos investigadores manifiestan que son inevitables los conflictos entre cónyuges en todos los aspectos de la vida diaria, y concuerdan que la clave está en el arte de pelear limpiamente o dicho de otro modo, el arte de resolver los conflictos; así lo refieren algunos autores como Rowe (2007); Chico (2010); Zambrano (2005) y Muñoz (2009). Se considera que la habilidad de satisfacer las necesidades emocionales y sexuales y el arte de resolver los conflictos son eslabones importantes para mantener un matrimonio saludable y estable, sin peligro de disfunciones y/o separaciones, tal y como lo manifiestan Núñez (2007); Van Pelt (2004); Vallejo, Sánchez y Sánchez, 2004 \& Chico (2010). Asimismo, algunos investigadores en temas de pareja afirman que las discusiones y los conflictos son los factores determinantes que impiden la satisfacción sexual (Rosberg \& Rosberg, 2006; Álvarez-Gayou, et al, 200).

En base a los resultados obtenidos se confirma la hipótesis general de la investigación, los estilos de negociación del conflicto influye en la intimidad de la pareja, como lo menciona también Larson, Hammond y Harper (1998). En cuanto a las hipótesis específicas se afirma que el estilo colaboración y evitación tienen relación con la satisfacción sexual, a diferencia de los estilos de acomodación y competencia. Al considerar las hipótesis, tenemos lo siguiente:

En primer lugar, con respecto al estilo de colaboración; las parejas adventistas que utilizan este estilo para resolver sus conflictos manifiestan una buena satisfacción sexual. Estadísticamente tiene una relación significativa de $(r=0.0441 ; p<0.01)$, confirmando la premisa de los autores como Rosberg y Rosberg (2006) y Álvarez- Gayou (1986): los cónyuges cuando resuelven los conflictos, considerando los puntos de vista de su cónyuge y encuentran una solución que beneficie a ambos, gozan de mayor satisfacción sexual. En este estilo colaborativo es muy evidente la existencia de una comunicación positiva entre los cónyuges, como lo indican las investigaciones. La comunicación positiva está asociada a una mayor satisfacción sexual y una buena calidad de relación con el cónyuge (Kirby, Baucom \& Peterman, 2005; Armenta y Díaz, 2006; Uribe, García y Ramírez, 2011). Asimismo, este estilo se caracteriza porque los cónyuges negocian constructivamente los conflictos, tienen una visión igualitaria, buscan una solución conjunta, como lo afirma Flores et al. (2004). Cuando los cónyuges integran las necesidades emocionales, espirituales, sociales y sexuales cumplen con el mandato bíblico "serán una sola carne" registrado en génesis 2:25 y Mateo 19:5 reflejando el diseño de Dios para el matrimonio, convirtiéndose la satisfacción sexual una acto de adoración al Creador, como lo mencionan diferentes autores cristianos entre ellos White (2009), Rosberg y Rosberg (2008), Ethridge (2004) y Nichol (1992). Al encontrar relación positiva entre el estilo de colaboración y la satisfacción sexual, la pareja experimenta mayor bienestar, erotismo, respeto, responsabilidad y comunicación; también podemos afirmar que indirectamente propicia la mejoría de la salud física y mental. La satisfacción sexual tiene beneficio cardioprotector, anticancerígeno, antidepresiva longevidad, entre otros, como lo ha investigado Arrondo (2008). El estilo de colaboración es el único estilo saludable y óptimo para una relación de pareja estable, mantiene un equilibrio en la relación en donde ambas partes ganan, ya que al integrar soluciones, maximizan los intereses de ambos (Vásquez, 2004; Flores et al., 2004).

En segundo lugar, con respecto al estilo de evitación; las parejas adventistas que usan este estilo para resolver sus problemas con su cónyuge también muestran que tiene influencia en la satisfacción sexual de manera positiva. Estadísticamente tiene $(r=0.235$, $p<0.05)$. Al usar este estilo, tienden aumentar la satisfacción sexual, a pesar de que es un estilo negativo de resolver el conflicto. La razón es que el estilo evitativo muestra dos caras. Es favorable cuando hay conflictos con el cónyuge y el otro insiste en continuar la discusión, entonces retirarse de la situación de forma diplomática y sin confrontaciones para evitar agresiones verbales y/o físicas es conveniente, como lo asevera Migali (2006). Lo desfavorable es evitar siempre el conflicto porque no resuelve los conflictos reales que pueden estar mermando la relación, así también lo afirma Flores et al., (2004) y Migali, (2006). Entonces las parejas adventistas que evitan el conflicto tienden a tener mayor satisfacción sexual, siempre y cuando resuelvan el conflicto, adoptando conductas diplomáticas como levantarse quedarse callado(a), levantarse y salir del cuarto con el fin de terminar la discusión; así mantienen un clima relacional estable propiciando confort al momento de la intimidad sexual.

Evitar el problema con otras personas que no sea la pareja es muy favorable, incluso evitar las discusiones entre los feligreses aumenta la espiritualidad y la unidad, como lo afirma White (1862). Sin embargo, con la pareja el estilo evitativo es solo una salida de emergencia, solucionamos el problema de manera 
superficial y no de forma profunda, no es la vía para afrontar los problemas reales. En una relación de pareja el estilo de evitación es una estrategia errónea de afrontar el conflicto, mencionan Shackelford y Buss (1997). Pero, de los estilos insalubres, este es el más sutil y es el que trae ventajas.

En tercer lugar, con respecto al estilo de acomodación; este estilo no tiene relación con la variable satisfacción sexual $(r=0.005, p>0.01)$. El hecho de ceder y satisfacer las necesidades emocionales y sexuales de la pareja, privándose de la propia, no se relaciona con la satisfacción sexual, ya que el acto sexual y la satisfacción sexual no concierne a una sola persona, sino a la pareja "hombre y mujer" donde buscan el deseo mutuo bienestar y plenitud durante la actividad sexual con el cónyuge como lo mencionan Carrobles, Gámez y Almendros (2011). En la satisfacción sexual la pareja evalúa el agrado de su vida sexual en relación a su cónyuge (Álvarez -Gayou et al., 2005).

Cuando uno de los cónyuges usan el estilo de acomodación actúa pasivamente, sacrificando sus propias necesidades entiende que "el que ama más da más" haciendo un "autosacrificio", de esta forma manifiesta sentimiento de inferioridad, como lo expresa Flores et al. (2004) no favoreciendo la satisfacción sexual. Sin embargo, la persona no lo percibe como negativo; Por el contrario, lo ve como un acto de generosidad, complace a su pareja para cuidar la relación matrimonial, tienen ideas irracionales como "la gente buena no pelea" "no tiene importancia" (Vásquez, 2004) o "los cristianos no pelean" influenciados por textos bíblicos mal entendidos, registrados en Lucas 6: 27-30, Efesios 5:21,22 que dicen: "Amad a vuestros enemigos, haced bien a los que os aborrecen", "bendecid a los que os maldicen, y orad por los que os calumnian". "Al que te hiera en una mejilla, preséntale también la otra; y al que te quite la capa, ni aun la túnica le niegues", "A cualquiera que te pida, dale; y al que tome lo que es tuyo, no pidas que te lo devuelva", "Someteos unos a otros en el temor de Dios", "Las casadas estén sujetas a sus propios maridos" entre otros textos. Esto conlleva que tomen una actitud pasiva acomodativa, queriendo decir que nunca vaya a ceder, sino que no debe regir en la relación de pareja.

Las parejas que usan el estilo de acomodación no tienen influencia en la satisfacción sexual; sino que hay otros factores mucho más predominantes que pueden estar mermando la intimidad sexual. Parish identifica los siguientes factores: dificultades en la práctica sexual, aspectos emocionales relacionados al deseo sexual, la salud física, conocimientos, actitudes, creencias y valores hacia la sexualidad (Santos et al., 2009). Y otros autores añaden aspectos como la ansiedad, estrés, baja motivación, falta de asertividad, falta de inteligencia sexual, adecuado manejo de tensión en la dinámica de la pareja, enfermedad, nivel de fatiga, baja autoestima, insatisfacción con la imagen corporal, falta de erotismo, cantidad de hijos y comunicación (Moral, 2010; Sánchez, Carreño, Martínez, Gómez, 2003; Escobedo, 2009; Zavala, 2009; Carrobles et al.,2011; Paredes y Pinto, 2009; Félix y Rodríguez, 2001; Armenta y Díaz, 2006; Masters et al, 1996; ÁlvarezGayou et al., 2005).

Finalmente, con respecto al estilo de competencia, cuando las parejas adventistas usan este estilo muestra que no tiene relación con la satisfacción sexual ( $r=$ $0.032, p>0.01$ ). Como ya mencionamos, una de las características más resaltante del estilo competencia es el uso del poder para imponer sus puntos de vista al cónyuge (Flores et al., 2004; Aguilera, 2007). El poder está definido como la habilidad para influenciar en el cónyuge y esta habilidad está determinada por estrategias o estilos de poder que usa (Rivera, 2000). Se ha realizado estudios encontrándose que hay muchas formas de ejercer el poder, ya sea directa o indirectamente; personal o impersonal, bilateral o unilateralmente, verbal o no verbal y que el grado de satisfacción que percibe cada uno de los miembros de la pareja tiene que ver con dichas estrategias y estilos que utilizan en su interacción (Rivera, 1996). Depende de cómo se perciba el poder, es negativo cuando la persona busca sus intereses a expensas del cónyuge avasallando de forma directa como lo corrobora Rivera (1996) y Félix y Rodríguez (2001); y el poder es sutilmente positivo cuando trata de convencer a su pareja con estrategias digno de ser admirado y con argumentos válidos (trabajo, estudio, finanzas, salud), tal como lo menciona Flores et al., (2004). Por ser ambivalente el poder en el estilo competitivo, no tiene relación con la satisfacción o insatisfacción sexual. El uso del estilo de competencia no tiene influencia en la intimidad sexual; sino que esta depende de otros factores más importantes similar al estilo de acomodación.

Por otra parte, cabe señalar algunos datos importantes propios de la investigación, como sigue:

En cuanto a los estilos más utilizados en la población adventista que vive en Lima Metropolitana está el estilo de acomodación y de competencia, seguidamente los estilos de colaboración y evitación, sin mucha variación en el porcentaje. En el distrito de Surquillo se usa el estilo de colaboración y evitación, mientras que el distrito de Villa María "A" se usa el estilo de acomodación y competencia. Estos resultados obtenidos es diferente a los estudios realizados en una 
población mexicana, donde el estilo más sobresaliente es el de colaboración seguido de acomodación y competencias, donde evidencian un proceso de transición entre el individuo y su sociocultura, así lo afirman Flores et al. (2004), Flores et al. (2005).

En cuanto a satisfacción sexual, el $50.7 \%$ de las parejas adventistas está en la categoría mala. Y en el distrito Villa María "A" experimentan mayor insatisfacción sexual en comparación al distrito de Surquillo. En otras palabras, la mayoría de la población estudiada tiene insatisfacción sexual; estos resultados son similares a los hallazgos encontrados por Zavala (2009). A diferencia de las parejas mexicanas que tienen satisfacción sexual en un nivel entre moderado y alto; porque dan importancia a los aspectos afectivos, consideran el amor y la comunicación para una buena relación sexual, así lo afirma Álvarez-Gayou et al. (2005). Por lo tanto, es conveniente evaluar los factores como el erotismo, bienestar, respeto y responsabilidad, estímulos sensoriales, afectividad, comunicación asertiva durante las relaciones sexuales; que influyen en la satisfacción sexual (Carrobles et al.,2011).

En cuanto a las diferencias por género, los resultados reflejan que las mujeres usan más el estilo de colaboración y los varones usan más el estilo de competencia. Las mujeres son más asertivas al usar el estilo de colaboración (Rojas, 2007; Honold, 2006). Tanto hombres y mujeres usan en segundo plano el estilo de acomodación, estos resultados son similares a las mujeres mexicanas cuando ceden y se sacrifican para llevar la relación en paz (Flores et al., 2004), también hay hombres que prefieren usar con frecuencia los estilos pasivos como lo encontraron Billings y Moos citado en Flores et al. (2004). Góngora (citado por Flores et al., 2004) señala que el matiz de los estilos de negociación del conflicto en el género está dada por la cultura. Por otro lado, encontramos que los hombres gozan de una buena satisfacción sexual en comparación con las mujeres, existiendo diferencias entre géneros como lo indica también Félix y Rodríguez (2001).
Y por último, se observó que el grado de escolaridad juega un papel importante; las personas que tienen instrucción superior optan el estilo de evitación y colaboración, mientras los que tienen estudios secundarios y primarios usan el estilo de competencia y acomodación; como también lo encontró Flores et al. (2004). Y a mayor escolaridad tienen mayor satisfacción sexual, estos hallazgos son similares a Honold (2006), Álvarez-Gayou et al. (2005), y Aguilar (2010).

\section{CONCLUSIONES}

De acuerdo a los datos y los resultados presentados en esta investigación, se llega a las siguientes conclusiones:

Existe asociación entre la variable dependiente (satisfacción sexual) y la variable independiente (estilos de negociación del conflicto), rechazando la hipótesis nula.

Hay una relación moderada altamente significativa entre el estilo colaboración y la satisfacción sexual en las parejas participantes. Es decir, aquellos que manifestaron emplear la colaboración como estilo para resolver los conflictos, presentaron también mayor satisfacción sexual.

Se encontró que no hay relación entre el estilo acomodación y satisfacción sexual en las parejas que participaron en el estudio. Es decir, que la satisfacción sexual no se vinculó al uso de este estilo.

En cuanto al estilo de evitación y la satisfacción sexual se refleja entre las variables una asociación baja pero significativa. Es decir, aquellas parejas que reportaron usar la evitación como estilo para resolver los conflictos presentaron mayor satisfacción sexual.

Finalmente el estilo de competencia y satisfacción sexual no tienen relación. Es decir, que la satisfacción sexual no se vinculó al uso de este estilo. 


\section{REFERENCIAS BIBLIOGRÁFICAS}

Aguilar, E. (2010). Satisfacción sexual femenina y su relación con la Dinámica familiar. [Tesis de especialización]. Universidad Michoacana de San Nicolás de Hidalgo, México.

Aguilera, V. (2007) Comprensión empática y estilos de negociación en la relación de pareja, herramientas de mediación. (Tesis de maestría, Universidad del País Vasco). Recuperado de http://psicologiarevista.99k. org/Comprension $\% 20$ Empatica $\% 20$ y $\% 20$ Estilos\%20de\%20Negociacion\%20en\%201a\%20 relacion $\% 20 \mathrm{de} \% 2$ pareja.pdf.

Álvarez-Gayou (1986). Sexoterapia integral. México: El Manual moderno

Álvarez-Gayou, J., Guerrero, B. y Ortega, S. (2001). Algunas reflexiones sobre la sexualidad erótica femenina. Archivos Hispanoamérica de Sexología 2 (1), 65-92.

Álvarez-Gayou, J., Honold, J. y Millán, P. (2005). Diseño de una escala autoaplicable para la evaluación de la satisfacción sexual en hombres $y$ mujeres mexicanos. Recuperado de http:// wmu.coaprendo.cl/elgg/raquel/files/2347/4810/ ejemplo3.pdf

Arana Z. M. (2002). Resolución de Conflictos Medioambientales en la Microcuenca del Río Porcón, Cajamarca 1993-2002 (Tesis inédita de maestría). Pontificia Universidad Católica del Perú, Perú.

Armenta, C. y Díaz, R. (2008). Comunicación y Satisfacción: Analizando la Interacción de Pareja. Psicología Iberoamericana, 16 (1), 23-27.

Arrondo, J. (2008). La sexualidad supone disfrutar, comunicarse, sentirse mejor y estar más sano. Revista Internacional Androl, 6(4), 260-264

Carrobles, J., Gámez, M., y Almendros, C. (junio, 2011) Funcionamiento Sexual, satisfacción sexual y bienestar psicológico y subjetivo en una muestra de mujeres españolas. Anales de Psicología, (27)1, 27-34

Chico, A. (2010). Lo que usted debe saber antes de dar el sí. Perú: Universidad Peruana Unión.

Ethridge, S. (2004). La batalla de cada mujer. Estados Unidos: Editorial Unilit.

Félix, M. y Rodríguez, A. (2001). Relación existente entre el estrés en la crianza y el número de hijos asociados a la satisfacción marital (tesis de licenciatura, Instituto Tecnológico de Sonora). Recuperado de http:// www. ciad.mx/desarrollo/publicaciones/Tesis $\% 20$ asesoradas/Licenciatura/33.pdf
Flores, M., Díaz, R., y Rivera, S. (2004) Validación psicométrica del inventario de negociación del conflicto en parejas de una subcultura tradicional. Ridep, 17 (1), 39- 55

Flores, M., Díaz, R., Rivera, S. y Chi, C. (Julio/ diciembre, 2005) Poder y negociación del conflicto en diferentes tipos de matrimonio. Enseñanza e investigación en psicología, 10(2), 338- 353

Gilbert, M. y Shmukler, D. (2000). Terapia breve con parejas. Un enfoque integrador. México: Editorial El Manual Moderno.

Honold, J. (2006) Estudio de correlación entre satisfacción sexual y asertividad sexual. Archivos Hispanoamericanos de Sexología, 12(2), 199 - 218

Instituto Nacional de Estadística e Informática. Nacimientos, defunciones, matrimonios y divorcios en el área metropolitana de Lima y Callao 20052006, 2007. Lima: Perú. Dirección Técnica de Demografía e Indicadores Sociales. Recuperado de http://www. inei.gob.pe/biblioineipub/bancopub/ Est/Lib0747/Libro.pdf

Kirby, J., Baucom, D. y Peterman, M. (2005). An Investigation of Unmet intimacy Needs in Marital relationships. Journal of Marital and Family Therapy, 31(4), 313-325.

Larson, J., Hammond, C. y Harper, J. (1998). Perceived Equity and Intimacy in Marriage. Journal of Marital and Family Therapy, 24(4), 487-506.

Levinger, G. y Pietromonaco, P. (1989). Conflict Style Inventory. Manuscrito no publicado. Universidad de Massachusetts, Amherst.

Masters, W., Johnson, V. y Kolodny, R. (1996). Eros, los mundos de la sexualidad. España: Editorial Grijalbo Mondadori, S.A.

Migali, J. (2006). Conflictos de pareja. Sanamente. Recuperado de http://www.sanamente.com.ar/t conflictos_pareja.html

Mirabal, D. (2003) Técnicas para manejo de conflictos, negociación y articulación de alianzas efectivas. Provincia, 1(10), 53 -71.

Moral, J. (2010). Satisfacción y frecuencia de las relaciones sexuales en parejas casadas. Ciencia UANL. Universidad Autónoma de Nuevo León, 8(1), 30-37.

Muñoz, A. (Marzo, 2009). Personas divorciadas: análisis de las características del proceso de ruptura. Intervención Psicosocial, 18(1), 65 - 74 
Nichol, F. (Ed.). (1992-1995). Comentario Bíblico Adventista del Séptimo Día (Tomo 1,5). Argentina: Asociación Casa Editora Sudamericana.

Núñez, M. (2007) Sexo y amor. Argentina: Asociación Casa Editora Sudamericana.

Ortiz, M. (marzo, 1999). La Salud Familiar. Revista Cubana de Medicina General Integral, 15(4), 439 -445 .

Paredes, J. y Pinto, B. (2009). Imagen corporal y satisfacción sexual. Ajayu, Universidad Católica Boliviana San Pablo. 7(1), 28-47.

Rivera, S. (1996). Estrategias y estilos de poder en la pareja mexicana. [Proyecto de Investigación]. Universidad Nacional Autónoma de México.

Rivera, A. (2000). Conceptualización, medición y correlatos de poder y pareja: Una aproximación etnopsicológica. [Tesis doctoral]. Universidad Nacional Autónoma de México.

Rivera, A., Díaz, R. y Sánchez, A. (2002). Revista de Psicología Social y Personalidad. 18 (1), 21-34.

Rosberg, G. y Rosberg, B. (2008). Las 5 necesidades sexuales de hombres y mujeres. Estados Unidos: Tyndale House Publisher, Inc.

Rowe, T. (05 de junio de 2007). El divorcio no siempre es inevitable; puede prevenirse, dice pastor adventista. Adventist News Network. Recuperado de http://news.adventist.org/archive/ articles/2007/06/05/north-america-divorce-notalways-inevit able-is-preventable-says-adventist-p

Sánchez, A. (2000). Validación empírica de la teoría bio-psico-socio-cultural de la relación de pareja. Tesis de doctorado no publicada. Facultad de Psicología. Universidad Nacional Autónoma de México.

Sánchez, C., Carreño, J., Martínez, S. y María, G. (2003). Comunicación marital y estilos de comunicación en mujeres con disfunción sexual. Perinatol Reprod Hum, (17) 91-101.

Santos, P., Sierra, J., Garcia, M., Martínez, A., Sánchez, A. y Tapia, M. (2009). Índice de satisfacción sexual (ISS): un estudio sobre su fiabilidad y validez. International Journal of Psychology and Psychological Therapy, 9(2), 259-273.

Uribe, I., García, M. y Ramírez, L. (2011). Relación entre estilos de comunicación y satisfacción sexual en estudiantes universitarios. Interamerican Journal of Psychology, 45(2), 157-167.

Vallejo, R., Sánchez, F. y Sánchez, P. (diciembre, 2004). Separación o divorcio: Trastornos psicológicos en los padres y los hijos. Revista de la Asociación Española de Neuropsiquiatría, (92), 91-110. doi: 10.4321/ S0211-5735200400 0400006

Van Pelt, N. (1985). Felices para siempre. Argentina: Asociación Casa Editora Sudamericana.

Van Pelt, N. (2001). Amor sin secretos. Argentina: Asociación Casa Editora Sudamericana.

Vásquez, W. (2004). Manual de medios alternativos de resolución de conflictos [Monografía]. Monografia. com. Recupe rado de http://www.monografias. com/ trabajos36/resolucion-conflictos/ resolucionconflictos.shtml

Veinticuatro municipios de Lima no cumplen con ley de divorcio rápido. (Octubre, 2010). Andina, Agencia Peruana de Noticias. Recuperado de http://www. andina.com.pe/ Espanol/ Noticia.aspx? $\mathrm{Id}=\mathrm{bHJj}$ QE0YJD0=

White, E. (1962). Primeros escritos. Argentina: Asociación Casa Editora Sudamricana

White, E. (2009). Mente, carácter y personalidad. Argentina: Asociación Casa Editora Sudamericana.

Wicker, C. (2000) Dumbfounded By Divorce: Survey Inspires Debate Over Why Faith isn't a Bigger Factor in Marriage. The Dallas Morning News. Recuperado de http://www.adherents. com/ largecom/baptist divorce.html

Zambrano, G. (2005) Unidos: hasta que la muerte nos separe. Ecuador: Editorial Cumbe.

Zavala, P. (2009) Inteligencia sexual, estrés y satisfacción sexual en adultos casados de Lurigancho Chosica. [Tesis de licenciatura]. Universidad Peruana Unión, Perú. 